\title{
Interactive Virtual Indoor Navigation System using Visual Recognition and Pedestrian Dead Reckoning Techniques
}

\author{
Chin Gee Low and Yunli Lee \\ Faculty of Science and Technology, Sunway University, \\ No. 5, Jalan Universiti, Bandar Sunway, 47500 Selangor, Malaysia \\ 03007531@imail.sunway.edu.my,yunlil@sunway.edu.my
}

\begin{abstract}
Finding a destination in an unfamiliar indoor environment requires cumbersome effort to refer to a physical floor plan or directory to locate the intended destination. With the advancements of mobile technologies, a navigational system using mobile computing devices such as mobile phone or tablet could aid users in locating the desired destination with ease. This paper presented an interactive virtual indoor navigation system which is developed for Sunway University campus. In order to provide an interactive and contextsensitive navigation platform, a hybrid solution has been proposed by blending the sensor capabilities on the mobile devices to work in an indoor environment. These sensors include utilizing the built-in accelerometer, compass and camera capabilities to create an interactive content of indoor navigation system using visual recognition and pedestrian dead reckoning for Augmented Reality (AR). Furthermore, user satisfaction and feedback survey have been collected for further improvement the proposed solution.
\end{abstract}

Keywords: Indoor Navigation System, Augmented Reality, Visual Recognition, Pedestrian Dead Reckoning, Vuforia SDK

\section{Introduction}

The recent advances in mobile technologies have seen the introduction of many innovative location-based services (LBS) concepts being implemented in mobile devices. This is often achieved through the use of components that are available in most of today's mobile devices which includes accelerometer, gyroscope, compass, camera, Global Positioning System (GPS), Bluetooth, and Wi-Fi. For instance, most LBS systems offered today uses GPS to provide the primary navigational capabilities while the use of Wi-Fi and cell tower triangulation is used to provide secondary or assisted geographical information to mobile users [1]. However, these systems exhibits limitations in an indoor environment or require additional installation of equipment and transmitters to function effectively. Henceforth, the keen interests in the area of indoor location-based applications have spurred new challenges in utilizing the onboard mobile device's sensors more efficiently and effectively to compensate for the inability of the GPS to work in an indoor environment [2].

This paper proposed an interactive virtual indoor navigation system for Android application with a low cost easy-to-use interactive navigational solution to aid users in locating their intended destinations in an indoor environment. It would act as a virtual guide that displays information of the Sunway University campus floor plan and layout via Augmented Reality (AR). AR refers to the technology whereby computer generated sensory inputs or information is overlaid onto our physical view of the world in real-time via the user's mobile device [3]. This provides a whole new level of interaction as information is seamlessly blended into our physical reality. This information could then be used to help users to their environment better as they are equipped with real-time information of their actual surroundings. With this, the proposed application is designed 
to leverage on the AR concept to overlay an interactive virtual 3D map of the campus environment together with its navigation path to guide the users to their intended destinations. The use of AR would also provide a seamless and intuitive experience to enhance a user's perception and understanding of their current environment.

Furthermore, we also examine the theoretical and technical aspect of creating an application that would provide an interactive guidance system to visitors of the campus as compared to conventional physical floor directory indicators. In order to achieve this goal, we leverage on using existing onboard sensors found commonly in today's mobile devices without the need for additional equipment or special infrastructures in the campus. As such, we design and develop an application to demonstrate the ability of using AR with image recognition techniques coupled with a Pedestrian Dead Reckoning (PDR) system to track the user's movement in an indoor environment. PDR is a technique that utilizes the accelerometer sensor to track the user's pattern of steps and with this, could also be used to perform step counts [4]. From the number of step count generated, we could then estimate the distance travelled by the user. Moreover, coupling the accelerometer with the compass sensor would provide the PDR system with the ability determine the heading and direction of the user.

A virtual 3D map is created in the Unity 3D software by referencing a floor plan of the campus obtained from the Sunway University Facilities Department. The virtual 3D map will then be overlaid on the actual live visual feed from the onboard camera. In order to identify and calibrate the current location of the user in the virtual 3D map, image recognition techniques are applied to initialize and identify locations in the Sunway University campus. The Vuforia SDK is an AR solution that provides the image recognition and processing capabilities for the proposed application [5]. Once the position and location of the user has been determined initially through visual recognition, the proposed application will continue to track the user's movement through a PDR system in the event that the Vuforia SDK is unable to recognize the next location or landmarks. As such, the PDR system is seen as a complementary solution to the Vuforia SDK.

The PDR system data is then relayed to the compass function found in the mobile device to enable the proposed application to distinguish the user's walking direction. With this, the user would be able to view in real-time the exact location and direction of their current position via the virtual 3D map that are displayed through AR on the mobile device. It has to be noted that the PDR system implemented in this project is susceptible to accumulated incremental errors and as such, would require a combination of the realtime visual tracking ability of the Vuforia SDK to enhance its accuracy. Furthermore, a calibrated PDR system has being implemented to provide a more robust solution in tracking the user's movement. Various techniques are explored and implemented to account for the cumulative errors and inaccuracies [6].

A mobile application prototype called SunMap+ [7] has been developed based on the proposed hybrid solution to evaluate the possibilities of indoor navigation using AR in the test environment of the Sunway University campus. This provides a whole new level of interaction as information is seamlessly blended into our physical reality to enhance the user's perception. This information could then be used to help users understand their environment better as they are equipped with real-time information of their actual surroundings. In order to gauge the level of acceptance of the SunMap+ application, a user satisfaction and feedback survey has been conducted. The survey is separated into three distinct categories covering the different aspects of the SunMap+ usage. It is designed to evaluate the SunMap+ prototype in terms of user friendliness, usability and navigation accuracy. Furthermore, the survey also takes into account of the implementation and usage of AR in the project to enhance information representation in providing navigational guidance. 


\section{Related Work}

There are various LBS systems on mobile devices that are available today which revolve around the use of a GPS to provide the necessary navigational functionalities and integrating it with AR. These systems mostly rely on the use of GPS to produce the desired outcome. However, these systems do not provide sufficient effectiveness in an indoor environment as they are prone to indoor interference. The GPS signal is unable to provide the necessary coverage in an indoor surrounding and is more suited for outdoor navigation [8]. As such, we proposed an application called SunMap+ and it is designed to provide an alternative solution towards indoor navigation based on an offline hybrid approach that utilizes visual image processing and object recognition capabilities coupled with a PDR system.

There are many applications to indoor navigations systems which includes personal virtual tour guide, indoor evacuation system and targeted mobile advertisements. We examine the use of various indoor positioning technologies that are currently being researched and applied in various scenarios. Table 1 provides a comparison between various indoor LBS applications in relation to SunMap+. The comparisons were made in terms of the applications' tracking and mapping capabilities and also its underlying indoor positioning technology used. Based on the Table 1, it could be seen that the use of AR coupled with the visual recognition approach provides various advantages over the existing indoor LBS solutions evaluated. Furthermore, the inclusion of the PDR system as an additional secondary tracking system in the SunMap+ provides increased reliability and robustness that is lacking in most of the existing indoor LBS solutions that we have evaluated.

\section{Table 1. Summary of the Characteristics and Attributes of the Indoor LBS Systems Evaluated}

\begin{tabular}{|c|c|c|c|c|}
\hline Application Name & $\begin{array}{l}\text { Proposed } \\
\text { SunMap+ }\end{array}$ & $\begin{array}{l}\text { Nintendo 3DS } \\
\text { Guide: Louvre } \\
\text { [9] }\end{array}$ & $\begin{array}{c}\text { CAViAR } \\
{[5]}\end{array}$ & $\begin{array}{c}\text { Roodin } \\
\text { [10] }\end{array}$ \\
\hline $\begin{array}{l}\text { LBS } \\
\text { Characteristics } \\
\text { and Attributes }\end{array}$ & $\begin{array}{l}\text { Visual } \\
\text { Recognition } \\
\text { + PDR }\end{array}$ & Wi-Fi & $\begin{array}{l}\text { Visual } \\
\text { Recognition } \\
\text { + PDR }\end{array}$ & $\begin{array}{c}\text { QR Code } \\
+ \text { PDR }\end{array}$ \\
\hline $\begin{array}{l}\text { Additional equipment, } \\
\text { transmitters or tracking tags } \\
\text { required }\end{array}$ & $\mathrm{NO}$ & YES & $\mathrm{NO}$ & YES \\
\hline $\begin{array}{l}\text { Susceptible to radio frequency } \\
\text { interference and attenuation }\end{array}$ & $\mathrm{NO}$ & YES & NO & NO \\
\hline Training data required & $\mathrm{NO}$ & YES & $\mathrm{NO}$ & $\mathrm{NO}$ \\
\hline Secondary tracking system & YES & $\mathrm{NO}$ & YES & YES \\
\hline Supports augmented reality & YES & $\mathrm{NO}$ & YES & NO \\
\hline $\begin{array}{l}\text { 3D mapping of the } \\
\text { environment }\end{array}$ & YES & $\mathrm{NO}$ & $\mathrm{NO}$ & $\mathrm{NO}$ \\
\hline Shortest path recommendation & YES & $\mathrm{NO}$ & YES & $\mathrm{NO}$ \\
\hline Turn-by-turn navigation & YES & $\mathrm{NO}$ & NO & NO \\
\hline
\end{tabular}

Aside from that, the use of a PDR system also gives an added advantage in providing a more generalized approach in detecting the user's movement without modification or knowledge of the environment as compared to the existing indoor LBS systems evaluated. This is because the PDR system works by calculating the distance travelled from a given starting position (localized through visual recognition) and track the user's movement through foot step detection [4]. As such, SunMap+ requires no specific additions to aid the accuracy of tracking the user in 
an indoor environment as compared to the other systems evaluated. For example, the LANDMARC system requires the use of RFID tags and base stations as part of the system setup. A study of the location and placement of RFID tags have to be coordinated to ensure that the positional information of each tag is known precisely to provide an accurate estimation of the location of the user [11]. The requirement to place these tags is seen as a limitation to the design principle behind the SunMap+ application. In this work, we proposed SunMap+ application requires that no additional equipment and changes are made to the existing environment of the campus.

Another major difference between the suggested indoor positioning approach introduced in the SunMap+ application and the radio frequency based systems is the susceptibility of radio signals to interference and attenuation in an indoor environment. Varying layouts architectures and moving objects usually will cause deterioration of the radio signals being emitted and would affect the accuracy to pinpoint the location of a user. This is usually caused by a number of factors which include reflection, refraction, diffraction, dead spots, and absorption of radio signals [12]. The SunMap+ approach, however, does not rely on the use of radio signals to track and locate the user as compared to the LANDMARC, and RADAR system. Instead, our approach of using visual recognition and a PDR system would resolve the instability issue of indoor radio signals propagation while providing accurate tracking ability of the user.

The SunMap+ application also differs from the Roodin application [10] which uses a QR code approach coupled with a PDR system. This is because the SunMap+ application leverages on real-world object recognition instead of constraining our system to the use of specially embedded codes. Instead of using QR codes, SunMap+ relies on the recognition of real world physical objects that are identifiable by the users in their surroundings. This is achieved by using the Vuforia SDK which provides the visual image processing capabilities that recognizes the natural features of an object. As such, the physical object in the real world would act as the marker of the location of the object. Hence, the experience is natural and intuitive for the user as it enhances their visual perception of the surrounding instead of locating QR codes that are placed in known locations. This is due to the fact that we are not limiting the user to scan particular QR codes to identify their location but enabling them to interact with their surroundings. With this, the use of SunMap+ provides a platform that encourages the creativity and freedom of creating location markers from surrounding objects as it is not constraint by the recognition of QR codes.

\section{System Overview}

The SunMap+ prototype was developed to demonstrate the feasibility and functionalities of the application in using the Vuforia SDK's visual recognition capabilities and AR features to localize the position of the user. Localization of the user has to be firstly initiated to provide the SunMap+ application with a reference point to anchor the user's starting position. Once the destination has been determined, the control of the application would flow to the Vuforia SDK which would continuously detect the surrounding area and determine the current location of the user using visual recognition. This is achieved by leveraging on the image recognition capabilities of the Vuforia SDK through the live camera feed which compares the feed to the pre-loaded unique images that was captured in the campus beforehand. A single image target is sufficient to identify the location of the user as the image target stores the coordinates of the particular location. The system architecture of the SunMap+ application is shown in Figure 1. 


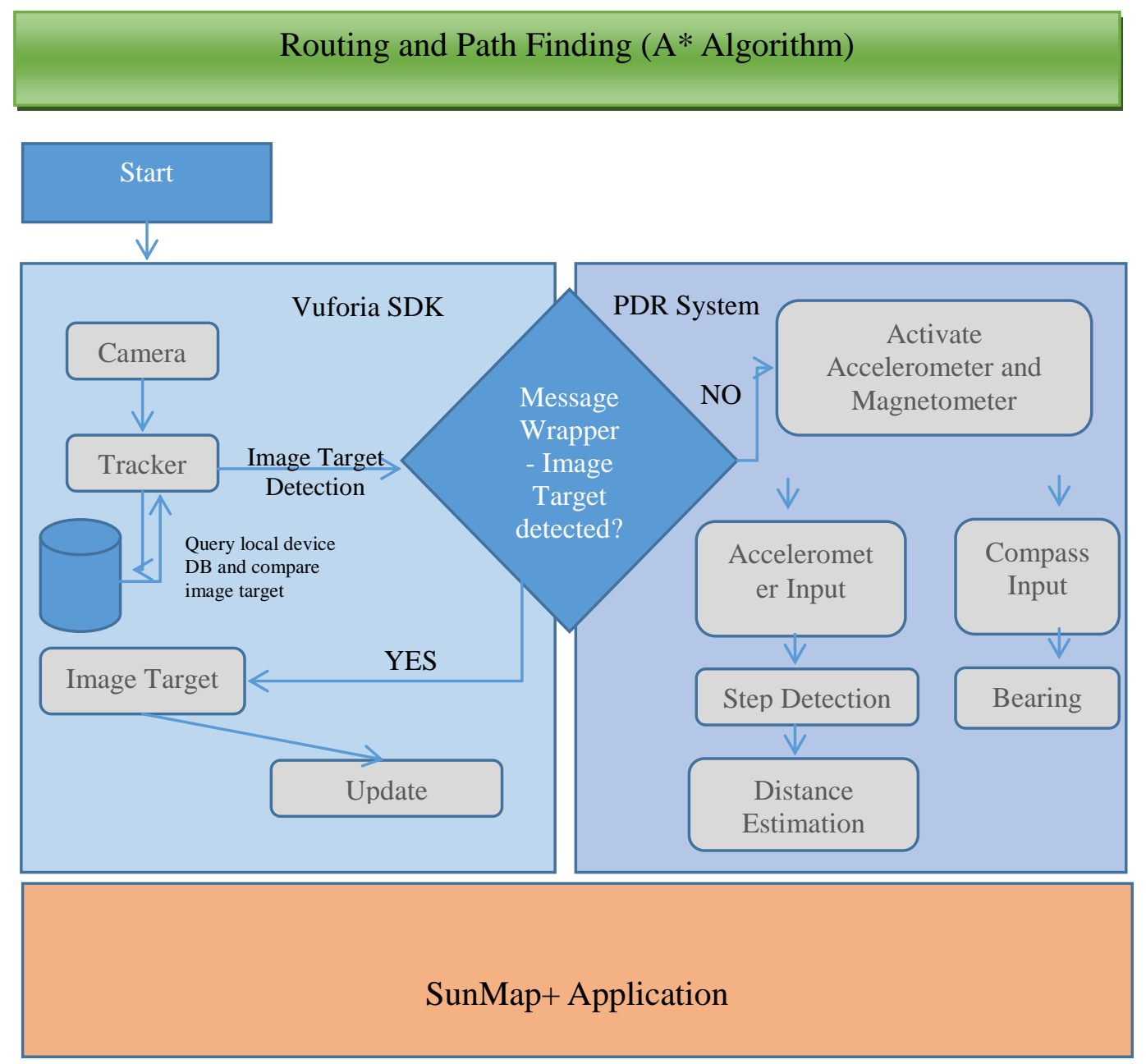

\section{Figure 1. Overall System Architecture of the Vuforia SDK and PDR System in the SunMap+ Application Environment}

In order to enhance the accuracy and tracking ability of the user's position, the PDR system has been implemented in the SunMap+ application. The PDR system would provide a complementary tracking feature of the user's location should the visual markers be not available for detection by the Vuforia SDK. This is achieved by recording the step counts of the user in a way that is similar to a pedometer. The step counter would then use this information to estimate the distance travelled by the user. The advantage of implementing such a system is that there are no additional changes that are required to be made to the existing infrastructure and allows the system to be infrastructure independent in order to track the user's movements. The PDR system is also coupled with the inbuilt compass sensor to provide directional and bearing information. This is important in determining the orientation of the user at any given time.

Once the location of the user has been identified, the information would be passed to the routing algorithm in the SunMap+ application. In our case, we will be using the $A^{*}$ algorithm [13] to determine the shortest path from our reference point to the intended destination. The routing information would be calculated and the shortest route will be displayed on the camera live feed. The information overlaid on the camera feed will indicate to the user the most suitable path that the user should take. As such, the overall system design requires the use of the sensing capabilities of the mobile device and is dependent on the user behavior to navigate to the destination. Re-routing will be calculated as soon as the position and heading of the original path is lost, upon which the SunMap+ application will then seek to provide an alternative route. 
The solution that has been proposed here differs from traditional indoor navigation systems of having to tag and mark the locations with QR or RFID tags. The SunMap+ application would be able to leverage on the Vuforia SDK provided by Qualcomm to perform image recognition in real-time of physical real world objects and deliver the information through the use of AR. The Vuforia SDK which acts as the enabler of the SunMap+ application in providing the image processing capabilities would be the primary localization method in providing the tracking and positional information of the user. If the image target is not found or could not be detected, the SunMap+ prototype would fall back to the pedometer function from our PDR system. Our PDR system here is designed to track the user movement and as well as the directional bearing of the user using the inbuilt compass sensor in the mobile device.

\section{Results and Discussion}

In this section, we discuss the results from the testing and simulation of the SunMap+ application conducted in the Sunway University campus. Two test plans have been created to simulate the reliability and performance of the SunMap+ application in terms of the accuracy of the Vuforia SDK and PDR system. The first test plan is initiated to measure the tracking ability of the SunMap+ application in relation to detecting an image target. On the other hand, the second test plan is used to measure the robustness and reliability of the PDR system in recording the step counts of the user. The data gathered would allow us to further improve the accuracy of each tracking mechanism respectively before evaluate it is integrated to form a fully functional indoor positioning system. Furthermore, a user satisfaction and feedback survey has been conducted to gauge the level of acceptance of the SunMap+ application.

\subsection{Vuforia SDK Localization Performance}

The fault tolerance ability of the Vuforia SDK to recognize image targets is included as part of the project test plan to ascertain the minimum visual percentage that is required to detect an image target. In other words, we seek to determine the maximum percentage of occlusion that the image target could tolerate before Vuforia SDK cease to detect the image target. We have designed the test plan by dividing an image target into four separate segments (Segment A, B, C and D) and occluding the image targets in proportion of $25 \%, 50 \%$ and $75 \%$. Each segment of the image target would then be tested for occlusion as the natural features of the image is not evenly distributed in every image. Table 2 shows the results of the occlusion test.

The data indicates that the Vuforia SDK has a fairly high fault tolerance ability of between $50 \%$ and $75 \%$. Based on the result, the Vuforia SDK is able perform reliably at detecting an image target with occlusion of up to $50 \%$. However, the image detection ability becomes less reliable and the augmentation starts to jitter when the image target is occluded at $75 \%$. Any occlusion above $75 \%$ results in the inability of the Vuforia SDK to detect the image target in our test plan. With this, we have to ensure that our design principles in the SunMap+ application takes into account of the fault tolerant ability of the Vuforia SDK. This is to ensure that the detection of image target is reliable and robust as we seek to simulate real-time occlusion that could happen in an actual environment. 


\section{Table 2. Result of the Vuforia SDK in Detecting an Image Target with Different Percentage of Occlusion}

\begin{tabular}{|c|c|c|c|c|}
\hline \multicolumn{5}{|c|}{ Fault Tolerance Ability } \\
\hline & \multicolumn{4}{|c|}{ Image Target Segment } \\
\hline Occlusion & A & B & C & D \\
\hline $0 \%$ occlusion & X & X & X & X \\
\hline $25 \%$ occlusion & $\mathrm{X}$ & $\mathrm{X}$ & $\mathrm{X}$ & $\mathrm{X}$ \\
\hline $50 \%$ occlusion & $\mathrm{X}$ & $\mathrm{X}$ & $\mathrm{X}$ & $\mathrm{X}$ \\
\hline $75 \%$ occlusion & $*$ & $*$ & $*$ & $*$ \\
\hline
\end{tabular}

Note: 'X' denotes successful detection of a particular segment, '*' denotes image target detection with less reliability and impairments

\subsection{PDR System Localization Performance}

In order to evaluate the accuracy of the PDR system, a survey has been set up with a total of six random participants of varying heights consisting of both the male and female population. This will provide an insight into the performance of the PDR system given that each participant with their unique attributes tends to have their unique stride length, walking speed and walking patterns. The test plan requires participants to walk five steps followed by a pause before continuing another five steps while holding the Samsung Galaxy Grand Duos mobile handset in a landscape orientation. Participants are required to walk a total of 10 steps and have the results from the PDR system compared against the actual step counts. The result of the survey is then recorded in Table 3 below. Based on the data collected, our PDR system managed to record an average step detection error rate of $8.3 \%$ from the 6 participants that took part in the survey. This indicates that the PDR system in our proposed solution is fairly accurate with a low mean error rate given that the approach does not rely on individualized calibration of each specific participant. The standard deviation of the survey is 1.1690 also indicates that the data points collected is close to the average value.

Table 3. Result from the PDR System Compared Against the Actual Step Counts

\begin{tabular}{|c|c|c|c|}
\hline Participant & $\begin{array}{c}\text { Avg. Measured } \\
\text { Steps }\end{array}$ & Actual Steps & Errors (\%) \\
\hline $\mathbf{1}$ & 8 & 10 & 20 \\
\hline $\mathbf{2}$ & 9 & 10 & 10 \\
\hline $\mathbf{3}$ & 10 & 10 & 0 \\
\hline $\mathbf{4}$ & 11 & 10 & -10 \\
\hline $\mathbf{5}$ & 10 & 10 & -10 \\
\hline $\mathbf{6}$ & 11 & 10 & -20 \\
\hline \multicolumn{3}{|c|}{ Average Error (\%) } \\
\hline
\end{tabular}

\subsection{User Satisfaction and Feedback}

The survey is separated into three distinct categories covering the different aspects of the SunMap+ usage. It is designed to evaluate the SunMap+ prototype in terms of user friendliness, usability and navigation accuracy. Furthermore, the survey also takes into account of the implementation and usage of AR in the project to enhance information representation in providing navigational guidance. A total of 10 participants were chosen randomly for this survey with a sample population consisting of six males and four females from varying age groups. 
As part of the survey, the participants were instructed to navigate to three different locations of their choice in the Sunway University campus using the SunMap+ application and provide their feedback on their experience thereafter. The feedbacks from the users were recorded immediately as soon as the users completed the task of navigating to their intended locations. The survey feedbacks were recorded on an electronic form that we have designed for this survey. The survey questionnaire consisted of a total of 12 questions with a combination of closed questions (multiple choice questions) and open questions (written comments for evaluation).

Based on the result in Figure 2, the bar chart illustrates the satisfaction level in terms of ease of use, usability and navigation accuracy of the SunMap+ application. The first aspect that we have surveyed involved the user friendliness and the ease of use of the SunMap+ user interface. From the feedback collected, $80 \%$ of the respondents agreed that the user interface of the SunMap+ is simple, intuitive and relatively easy to use. Majority of the users do not have trouble navigating through the menus and options in the SunMap+ application. However, the survey indicated that $20 \%$ of the users found the application unsatisfactory due to the font size and button size used is too small. This is certainly an area of improvement that should be noted in order to improve the user experience in the next version of the SunMap+ application.

Another improvement that should be taken into consideration is the slow loading times required when changing screens as noted by another $30 \%$ of the survey population. The considerable long loading time taken to change screen is due to the extensive path calculation and routing process that is running in the background which will be addressed in the next version of the SunMap+ application. From the written evaluation of the survey feedback, we have also noted that $20 \%$ of the users found that the color scheme used in the application to be dull and not contemporary.

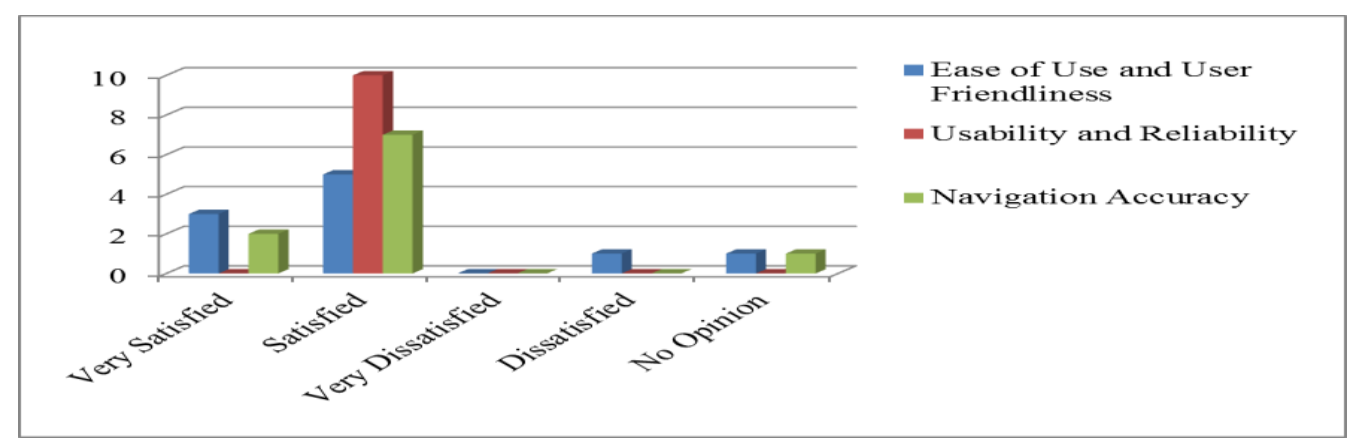

Figure 2. The Satisfaction Survey Result is Measured in Terms of the Ease of Use, Usability and Navigation Accuracy of the SunMap+ Application

The survey conducted also seeks to measure the usability and reliability of the SunMap+ application in providing navigational guidance to the users. Based on the feedback result, all the survey participants indicated that they would recommend the SunMap+ application to their friends and peers as they found the application to be helpful in shortening the time taken in locating their destination. A notable feedback received to further improve the usability and reliability of the SunMap+ application is to include a search function on the locations that are listed in the SunMap+ application. The current version of the SunMap+ does not include this feature as the users are required to scroll through a list of available locations that have been pre-determined.

Some other suggestions received to further improve the SunMap+ application include supporting more POIs in the campus and providing more information on the locations that are listed in the SunMap+ application. We have also received feedback to support different orientations in holding the mobile device instead of the pre-determined landscape orientation. This is mainly caused by the fact that holding the phone in a 
vertical orientation requires the use of both hands as the Samsung Galaxy Grand Duos is rather large in size and could rather prove to be an inconvenience to the users. Another feedback received to improve the SunMap+ application is that cross platform support is provided.

Based on analysis of the survey result, all participants agreed that the SunMap+ application has successfully achieved its objective in improving information representation of navigational guidance through the use of AR. Respondents shared their views that the use of AR enables them to visualize the navigation path with ease as the information overlaid on the camera live feed is relevant and context sensitive in nature. In addition to that, the $3 \mathrm{D}$ view of the virtual map is seen as an important element in improving the users' understanding and perception of the campus layout. Furthermore, majority of users found that the use of AR in the SunMap+ application enabled them to navigate their environment with more confidence and perceive their environment better in locating their destination.

From the survey result gathered, we could conclude that the SunMap+ application managed to achieve its objective in providing a cost effective indoor positioning solution that is reliable and accurate using existing sensors that are available in most of today's mobile devices. The SunMap+ application also successfully achieved its objective in using AR to enhance the usability of the system as it provides users with a better perspective and understanding of their environment. Furthermore, the SunMap+ application is also well accepted due to its ease of use and user friendliness. By leveraging on the AR approach, we could minimize the use of menus and buttons on the interface as gestures and hand motions are used instead.

\section{Conclusion}

The proposed SunMap+ prototype has demonstrated that it is possible to use visual recognition technique complemented by a PDR system to achieve a high level degree of accuracy to track a user's movement and location using a mobile device. The use of visual recognition as an absolute positioning mechanism in an indoor environment also helped address the incremental errors generated from the PDR system. Furthermore, we also seek to demonstrate the ability of the SunMap+ application to function as a virtual navigator for visitors to the Sunway University campus who might not be familiar with their surroundings. The SunMap+ prototype was successfully implemented as a costeffective solution and provides a user-friendly virtual navigation system to visitors of the campus. The AR approach being implemented in the SunMap+ prototype also enhances the visual representation of navigational information that could be easily perceived and understood by visitors of the campus. Henceforth, indoor positioning technologies and the use of AR is seen as an area with great research potential that would yield many benefits. All in all, the SunMap+ prototype is designed to function as an indoor positioning ubiquitous application that could be applied in our daily lives and routines. The same concept could be applied to various applications and should not be solely confined to the existing usage suggested in this work. As such, AR technology could one day be seen as the linkage to bridge the divide between the physical real world and its digital counterpart to provide an integrated solution to having intelligent and contextsensitive information.

\section{Acknowledgements}

This work was supported by Sunway University and we thank all users participating in the experiment. 


\section{References}

[1] V. Otsason, A. Varshavsky, A. LaMarca and E. Lara, "Accurate GSM Indoor Localization", Proceedings of $7^{\text {th }}$ International Conference, UbiComp (2005), September 11-14, Tokyo, Japan.

[2] J. H. Choo, S. N. Cheong, Y. L. Lee and S. H. Teh, "I ${ }^{2}$ Navi: An Indoor Interactive NFC Navigation System for Android Smartphones", Proceedings of the World Academy of Science, Engineering and Technology, vol. 72, (2012).

[3] D. Schmalstieg, T. Langlotz and M. Billinghurst, "Augmented Reality 2.0 in Virtual Realities", Edited S. Coquillart, et al., Springer, Vienna, (2011), pp. 13-37.

[4] A. R. Pratama and R. Hidayat, "Smartphone-based Pedestrian Dead Reckoning as an indoor positioning system", Proceeding of IEEE in International Conference on System Engineering and Technology (ICSET) IEEE, (2012) September 11-12, Bandung, Indonesia.

[5] B. Delail, L. Weruaga and M. Zemerly, "CAViAR: Context Aware Visual Indoor Augmented Reality for a University Campus", Proceeding of IEEE International Conferences on Web Intelligence and Intelligent Agent Technology, (2012) December 4-7, Macau, Hong Kong.

[6] M. Kalkusch, T. Lidy, M. Knapp, G. Reitmayr, H. Kaufmann and D. Schmalstieg, "Structured Visual Markers for Indoor Pathfinding", Proceedings of the First IEEE International Workshop on ARToolKit (ART02), (2002).

[7] C. G. Low and Y. L. Lee, "SUNMAP+: An Intelligent Location-Based Virtual Indoor Navigation System using Augmented Reality", The IET International Conference on Frontiers of Communications, Networks and Applications (IET ICFCNA), (2014) November 3-5, Kuala Lumpur, Malaysia.

[8] C. Randell, C. Djiallis and H. Muller, "Personal Position Measurement Using Dead Reckoning", in Proceedings of the Seventh International Symposium on Wearable Computers, C. Narayanaswami, Ed. IEEE Computer Society, (2003) October 21-23, New York, USA.

[9] "The Verge", The Louvre now offering Nintendo 3DS audio guides with built-in maps, (2012).

[10] A. Serra, D. Carboni and V. Marotto, "Indoor pedestrian navigation system using a modern smartphone", In Proceedings of MobileHCI2010. ACM Press, (2010) September 7-10, Lisboa, Portugal.

[11] A. Ye, Y. Ling, L. Xu and X. Yang, "An Improved RFID-Based Localization Algorithm for Wireless Sensor Networks", International Journal of Distributed Sensor Networks, (2013).

[12] J. Ahn and R. Han, "An indoor augmented-reality evacuation system for the Smartphone using personalized Pedometry”, Human-centric Comput Inf Sci, Springer, vol. 2, no. 1, (2012), pp. 18.

[13] X. Cui and H. Shi, "A based path finding in modern computer games", International Journal of Computer Science and Network Security, vol. 11, no. 1, (2011), pp. 126.

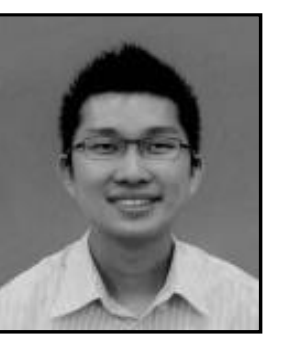

Authors

Chin Gee Low, graduated in 2007 with a Bachelor of Business and Commerce (Banking and Finance) and Bachelor of Business Systems double degree from Monash University. Upon graduation, he joined IBM as a Software Engineer specializing in disaster recovery solutions and data storage products, before moving on to Royal Dutch Shell to work in the area of project management. He is currently an IT Consultant with IBM's Systems \& Technology Group (STG), a position he has held since February 2012. His current role involves providing consulting services in technologies relating to Cloud Computing, Virtualization, IBM Power Systems and IBM Disaster Recovery solutions.

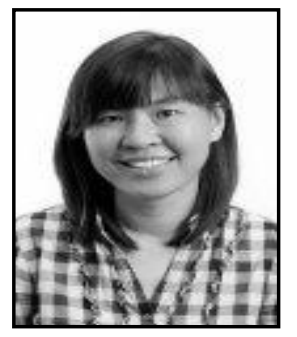

Yunli Lee, received her Ph.D. in Engineering (Digital Media) from Soongsil University, Seoul, Korea, 2009, Master in Engineering (Software) from Dongseo University, Busan, Korea, 2004 and Bachelor degree of IT (Hons) Software Engineering from Multimedia University, Selangor, Malaysia, 2002. She is currently a senior lecturer in Faculty of Science and Technology, Sunway University, Malaysia. Her research interests include pattern recognition, computer vision and graphics, image processing and human-machine interaction. 